\title{
Alterações físico-químicas em frutos de melão rendilhado sob aplicação de bioestimulante
}

\section{Physical-chemical changes in muskmelon fruits under biostimulant applications}

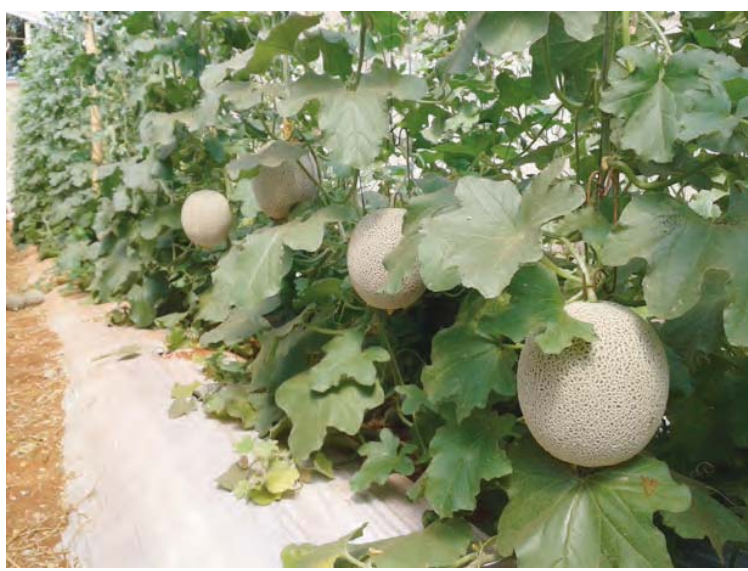

EDUARDO PRADI VENDRUSCOLO1,3

RODRIGO SOUZA RABELO²

LUIZ FERNANDES CARDOSO CAMPOS

ANGÉLICA PIRES BATISTA MARTINS' ${ }^{1}$

LEANDRA REGINA SEMENSATO

ALEXSANDER SELEGUINI

\section{RESUMO}

O alto valor de mercado dos frutos de meloeiro rendilhado permite que novas tecnologias sejam aplicadas a sua produção, visando à melhoria das qualidades fitotécnicas e tecnológicas de pós-colheita. Uma tecnologia a ser explorada é a utilização de bioestimulantes comerciais. O trabalho teve como objetivo avaliar a ação de bioestimulante, aplicado diretamente sobre frutos de meloeiro Cantaloupe, sobre diferentes características dos mesmos. Foram utilizadas diferentes concentrações de bioestimulante $(0,5$, 10 , 15, e $20 \mathrm{~mL} \mathrm{~L}^{-1}$ ), aplicadas quando o fruto se encontrava com tamanho menor ou igual a um (1) centímetro de diâmetro. No momento da colheita foi avaliada a massa dos frutos (g), as espessuras do epicarpo $(\mathrm{mm})$, do mesocarpo $(\mathrm{mm})$ e do halo verde do mesocarpo $(\mathrm{mm})$, o teor de sólidos solúveis ( $\left.{ }^{\circ} \mathrm{Brix}\right)$ e a razão entre circunferência longitudinal e transversal. Observou-se que a aplicação de concentrações crescentes do bioestimulante, até a dose máxima de $20 \mathrm{~mL} \mathrm{~L}^{-1}$, diretamente sobre o fruto, elevou o teor de sólidos solúveis até $11,5^{\circ}$ Brix. Desta forma, indica-se para o meloeiro em fase inicial de desenvolvimento, a aplicação de bioestimulante até a concentração de $20 \mathrm{ml} \mathrm{L}^{-1}$, diretamente sobre seus frutos.

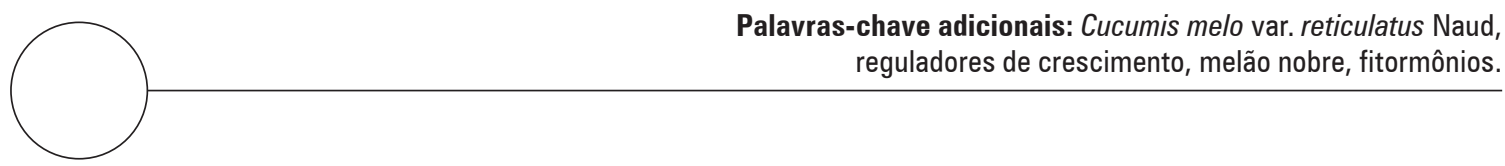

\footnotetext{
Escola de Agronomia, Universidade Federal de Goiás, Goiânia-GO (Brasil). ORCID Vendruscolo, E.P.: 0000-00023404-8534; ORCID Campos, L.F.C.: 0000-0001-5171-5194; ORCID Martins, A.P.B.: 0000-0002-1698-592X; ORCID Semensato, L.R.: 0000-0002-3961-6051; ORCID Seleguini, A.: 0000-0002-5762-9278

2 Uni-Anhanguera, Centro Universitário de Goiás, Goiânia (Brasil). ORCID Rabelo, R.S.: 0000-0002-8152-3465

Autor para correspondência. agrovendruscolo@gmail.com
} 


\begin{abstract}
The high market value of muskmelon fruit fosters new technologies for its production, aimed at improving plant quality and postharvest technological qualities. The use of commercial biostimulants was explored. This study aimed to analyze the action of biostimulant concentrations applied directly on Cantaloupe melon fruits on different characteristics. The biostimulant was applied at different concentrations $\left(0,5,10,15\right.$, and $\left.20 \mathrm{~mL} \mathrm{~L}^{-1}\right)$ when the fruits were smaller than or equal to one (1) $\mathrm{cm}$ in diameter. At harvest time, the fruits' weight $(\mathrm{g})$, peel thickness $(\mathrm{mm})$, mesocarp $(\mathrm{mm})$ and green mesocarp halo $(\mathrm{mm})$, soluble solids ( ${ }^{\circ}$ Brix) and ratio of longitudinal and transversal circumferences were evaluated. The application of increasing concentrations of biostimulant, up to the maximum dose of $20 \mathrm{~mL} \mathrm{~L}^{-1}$, directly on the fruits increased the soluble solids content to up to $11.5^{\circ} \mathrm{Brix}$. Therefore, biostimulant concentrations of up to $20 \mathrm{~mL} \mathrm{~L}^{-1}$ are recommended for applications directly on melon fruits in the initial stage of development.
\end{abstract}

Additional key words: Cucumis melo var. reticulatus Naud, growth regulators, noble melon, phytohormones.

Data de recepção: 16-01-2017 Aprovado para publicação: 30-09-2017
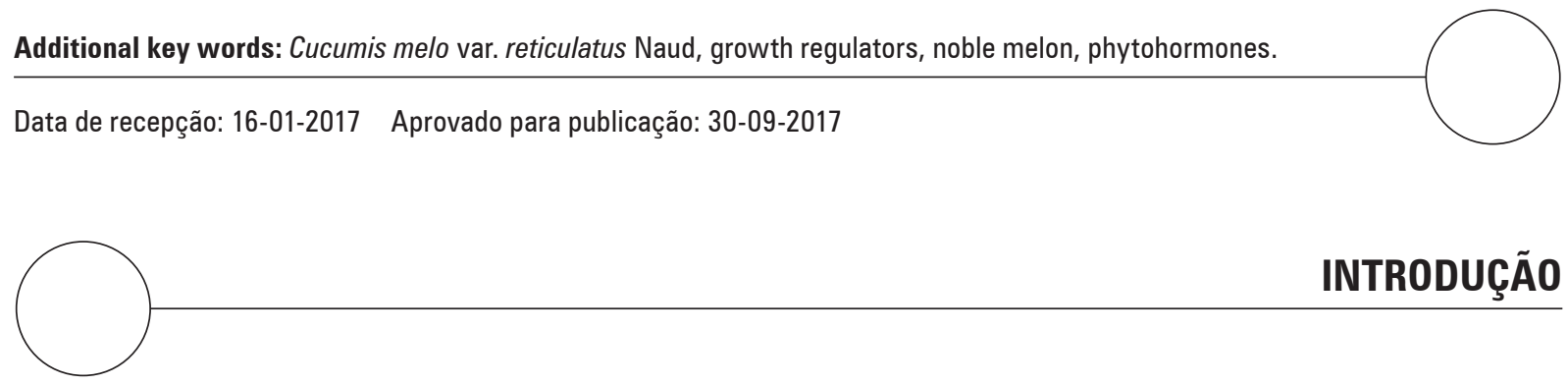

Nos últimos anos a produção de melões no Brasil tem crescido expressivamente, apresentando um aumento de $250.000 \mathrm{Mg}$ no período de 2004 a 2014, em uma área de aproximadamente 22.000 ha, com uma média de produtividade anual de $25,1 \mathrm{Mg} \mathrm{ha} \mathrm{M}^{-1}$. O Nordeste caracteriza-se como a principal região produtora e é responsável por cerca de 95\% de toda a produção nacional (IBGE, 2016), mesmo a cultura tendo boa aptidão para ser produzida em praticamente todo o território nacional.

Popularmente conhecidos como melão japonês ou Cantaloupe, os melões nobres fazem parte do grupo de melões rendilhados (Cucumis melo var. reticulatus Naud.), do grupo cantalupensis. Esses melões têm despertado grande interesse por apresentar qualidade superior aos demais melões tradicionais quanto ao aroma, polpa com coloração mais atraente e maior teor de sólidos solúveis (Medeiros et al., 2007).

alto valor de mercado dos frutos de meloeiro nobre permite que novas tecnologias sejam aplicadas a sua produção, visando à melhoria das qualidades fitotécnicas e tecnológicas de pós-colheita. Uma tecnologia a ser explorada é a utilização de bioestimulantes comerciais. Estes produtos vêm sendo utilizados para diferentes culturas, obtendo-se resultados variados dependendo da espécie em estudo (Izidório et al., 2015; Tavares et al., 2015; Vendruscolo et al., 2015). termo bioestimulante refere-se à mistura de dois ou mais reguladores vegetais ou de biorreguladores com outros produtos (Vieira e Castro, 2001). Estes compostos são aplicados de forma exógena nas plantas visando alterações nos processos vitais e estruturais, melhorias da produtividade e da qualidade do produto a ser comercializado e também dos manejos culturais (Chiavegato et al., 2007).

Apesar da quantidade considerável de estudos acerca da aplicação de bioestimulantes, informações sobre a utilização destes produtos diretamente sobre os frutos e, consequentemente, sobre o seu efeito no desenvolvimento destes, são escassos. Assim, a geração de novas informações de caráter técnico-científicas possui considerável significância.

Portanto, objetivou-se avaliar a ação de bioestimulante, aplicado diretamente sobre frutos de meloeiro rendilhado, sobre diferentes características físico-químicas dos mesmos.

\section{MATERIAL E MÉTODOS}

○ estudo foi realizado em Goiânia, Goiás. $\bigcirc$ município situa-se na região central do Estado, tendo a área experimental a localização de 16³5'55,2" S e 49¹6'39,1"W e altitude de $750 \mathrm{~m}$. O cultivo foi 
feito em ambiente protegido previamente construído na Escola de Agronomia da Universidade Federal de Goiás. Foi utilizada uma estufa, modelo arco, possuindo dimensão de $21 \times 7 \mathrm{~m}$ e altura de $4 \mathrm{~m}$, coberta com lona transparente e possuindo em suas laterais tela antiafídica branca. Durante o período de condução do estudo a umidade relativa do ar média foi de $66,7 \%$ e a temperatura média de $24,9^{\circ} \mathrm{C}$.

O solo presente na área experimental é classificado como Latossolo Vermelho distroférrico (Santos et al., 2013) e apresentou as seguintes características químicas na camada de 0-0,20 m: 0,7\% de M.O.; pH 4,6 $\left(\mathrm{CaCl}_{2}\right) ; 3,5 \mathrm{mg} \mathrm{dm}^{-3} \mathrm{P}$ (Mehlich); 131,0 $\mathrm{mg} \mathrm{dm}^{-3} \mathrm{~K}$; 2,0 $\mathrm{cmol}_{\mathrm{c}} \mathrm{dm}^{-3} \mathrm{Ca} ; 0,81 \mathrm{cmol}_{\mathrm{c}} \mathrm{dm}^{-3} \mathrm{Mg} ; 2,5 \mathrm{cmol}_{\mathrm{c}} \mathrm{dm}^{-3}$ $\mathrm{H}+\mathrm{Al} ; 0,0 \mathrm{cmol}_{\mathrm{c}} \mathrm{dm}^{-3} \mathrm{Al} ; 5,6 \mathrm{cmol}_{\mathrm{c}} \mathrm{dm}^{-3}$ CTC; $\mathrm{m} \%$ igual a 0,0; V\% igual a 55,7 (Donagemma et al., 2011).

Foi utilizado o delineamento em blocos ao acaso, com cinco tratamentos, formados pela variação da concentração de bioestimulante $\left(0,5,10,15\right.$ e $\left.20 \mathrm{~mL} \mathrm{~L}^{-1}\right)$, em cinco repetições. Cada parcela foi constituída por uma planta, com um fruto. $\mathrm{O}$ bioestimulante foi aplicado, com o auxílio de um pincel, diretamente sobre os frutos com tamanho igual ou inferior a um centímetro de diâmetro e reaplicado sete dias após.

Foi utilizado o bioestimulante comercial Stimulate® que possui em sua composição três reguladores vegetais nas seguintes proporções: $0,09 \mathrm{~g} \mathrm{~L}^{-1}$ de cinetina (citocinina), 0,05 $\mathrm{g} \mathrm{L}^{-1}$ de ácido giberélico (giberelina), $0,05 \mathrm{~g} \mathrm{~L}^{-1}$ de ácido indolbutiríco (auxina) e 99,98\% de ingredientes inertes (Agrofit, 2017).

O preparo da área de plantio constituiu da correção do $\mathrm{pH}$ para $6,0 \mathrm{com}$ a aplicação de calcário $(30 \%$ $\mathrm{CaO}, 18 \%$ MgO e PRNT de 100\%), conforme recomendação de Sobrinho et al. (2008). Posteriormente à realização da calagem foi feito o revolvimento do solo com enxada rotativa acoplada a um micro trator de rabiça. $\bigcirc$ manejo de plantas espontâneas foi feito por meio de capinas manuais, conforme a necessidade.

As mudas de meloeiro nobre foram obtidas por meio da semeadura em bandejas preenchidas com substrato composto por três partes de substrato comercial turfoso para uma de esterco bovino curtido, onde permaneceram até atingirem altura aproximada de 0,15 $\mathrm{m}$, sendo em seguida transplantadas para os canteiros, espaçadas $0,35 \mathrm{~m}$ entre si e tutoradas na forma de espaldeira. As linhas de plantio, na porção central do canteiro, foram espaçadas em $0,80 \mathrm{~m}$ entre si.
A irrigação foi realizada por meio de fitas gotejadoras, com gotejadores espaçados em 0,20 m entre si, que foram instaladas nas linhas de plantio e acionadas conforme a necessidade das plantas, observando-se as condições ambientais e o estágio de desenvolvimento (Sobrinho et al., 2008).

A adubação de plantio foi realizada no momento da confecção dos canteiros, utilizando-se três litros de esterco e $80 \mathrm{~kg} \mathrm{ha}^{-1}$ de fósforo, tendo como fonte o fertilizante termofosfato "Yoorin Master" (Yoorin, Poços de Caldas-MG, Brasil). As adubações de cobertura foram feitas com base nas recomendações de Sobrinho et al. (2008), aplicando-se $10 \mathrm{~kg} \mathrm{ha}^{-1} \mathrm{de}$ nitrogênio (ureia) e $5 \mathrm{~kg} \mathrm{ha}^{-1}$ de potássio (cloreto de potássio) semanalmente, via água de irrigação.

Na etapa final, no momento da colheita, foi avaliada, em laboratório, a massa dos frutos (g) por meio de pesagem em balança digital W15 (Welmy, Sta. Bárbara d'Oeste-SP, Brasil) com precisão de $5 \mathrm{~g}$, as espessuras do epicarpo ( $\mathrm{mm})$, do mesocarpo $(\mathrm{mm})$, do halo verde do mesocarpo ( $\mathrm{mm}$ ) e o diâmetro da cavidade interna do fruto, obtidos com paquímetro digital (Metrotools, São Paulo, SP, Brasil) e o teor de sólidos solúveis ('Brix) por meio da leitura em refratômetro manual RTA-50 (Instrutherm, São Paulo-SP, Brasil). Também foram mensuradas as circunferências longitudinal e transversal, para obtenção da razão entre as medidas.

Os dados experimentais foram submetidos à análise de variância e as médias do fator quantitativo (concentrações de bioestimulante) foram avaliadas pela análise de regressão a 5\% de probabilidade.

\section{RESULTADOS E DISCUSSÃO}

Observou-se que a aplicação de doses crescentes de bioestimulante não afetou as variáveis de massa de frutos, razão entre circunferência longitudinal e transversal, as espessuras de epicarpo, mesocarpo e halo verde do mesocarpo e diâmetro da cavidade interna do fruto. No entanto, estimulou o aumento dos teores de sólidos solúveis (Tab. 1).

Para todos os tratamentos, os frutos obtidos apresentaram alta qualidade física. A média da massa por fruto obtida foi de $1.251,44 \mathrm{~g}$, também se verificou que os frutos apresentavam formato esférico, adequado grau de maturidade e pequena cavidade interna, características favoráveis à comercialização, ao transporte e 
Tabela 1. Resumo da análise de variância e quadrados médios das variáveis de massa de frutos, razão entre circunferência longitudinal e transversal (CL/CT), espessuras de epicarpo, mesocarpo e halo verde do mesocarpo, diâmetro da cavidade interna do fruto e sólidos solúveis (SS) de frutos de melão Cantaloupe submetidos à aplicação de diferentes doses de bioestimulante.

\begin{tabular}{|l|l|c|c|c|c|c|c|c|}
\hline \multirow{2}{*}{ FV } & \multirow{2}{*}{ GL } & \multicolumn{7}{c|}{ Quadrados médios } \\
\cline { 3 - 9 } & & Massa & CL/CT & Epicarpo & Mesocarpo & Halo verde & Cavidade & SS \\
\hline Doses & 4 & $31.631,24^{\text {ns }}$ & $0,0005^{\text {ns }}$ & $0,0127^{\text {ns }}$ & $18,5216^{\text {ns }}$ & $0,7537^{\text {ns }}$ & $25,9343^{\text {ns }}$ & $5,0786^{*}$ \\
\hline Bloco & 4 & $107.756,14$ & 0,0009 & 0,0353 & 51,9960 & 1,6221 & 33,2884 & 3,3226 \\
\hline Erro & 16 & $117.497,54$ & 0,0010 & 0,0262 & 49,4536 & 1,2953 & 18,7506 & 1,1839 \\
\hline CV \% & & 27,39 & 3,00 & 21,98 & 21,67 & 21,3 & 15,48 & 10,55 \\
\hline Média geral & & $1.251,44 \mathrm{~g}$ & 1,04 & $0,74 \mathrm{~mm}$ & $32,45 \mathrm{~mm}$ & $5,34 \mathrm{~mm}$ & $27,98 \mathrm{~mm}$ & $10,31^{\circ} \mathrm{Brix}$ \\
\hline
\end{tabular}

FV: fator de variação; GL: graus de liberdade; CV: coeficiente de variação; ${ }^{\text {ns }} \mathrm{e}^{*}=$ Não significativo e significativo a $5 \%$ de probabilidade pelo teste de regressão, respectivamente.

ao consumo in natura (Medeiros et al., 2015). Porém, apenas frutos tratados com concentrações de 15 e $20 \mathrm{~mL} \mathrm{~L}^{-1}$ de bioestimulante apresentaram teores de sólidos solúveis acima de $10^{\circ}$ Brix, teor exigido para a comercialização deste tipo de melão (Sales Junior et al., 2004).

Obteve-se aumento dos teores de sólidos solúveis com a utilização de concentrações crescentes de bioestimulante, até $20 \mathrm{~mL} \mathrm{~L}^{-1}$, culminando em um teor máximo de aproximadamente $11,5^{\circ}$ Brix (Fig. 1). Corroborando com os resultados obtidos, a aplicação combinada de ácido giberélico (1.000 $\mathrm{mg} \mathrm{L}^{-1}$ ) e ácido naftaleno acético (450 $\left.\mathrm{mg} \mathrm{L}^{-1}\right)$, direcionada aos frutos partenocárpicos de atemóia, propiciaram aumento do teor de sólidos solúveis (Pereira et al., 2014).

O resultado está relacionado com o aumento da atividade de divisão e alongamento celular, propiciada

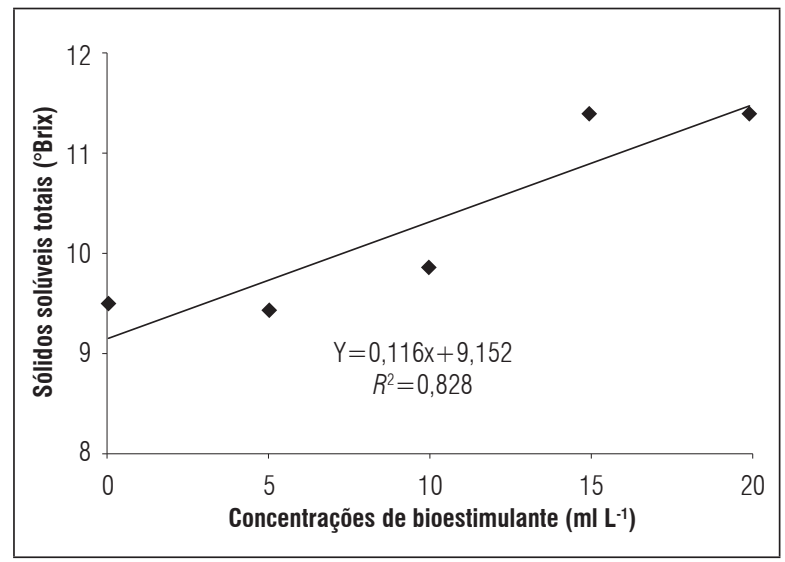

Figura 1. Teores de sólidos solúveis em relação às concentrações de bioestimulante. pela ação dos hormônios que compõem o bioestimulante (Taiz et al., 2017). Desta forma, eleva-se a capacidade dos frutos de atuarem como fortes drenos de compostos fotoassimilados, aumentando a fixação do fruto à planta (Talón et al., 1998; Prado et al., 2007) e, possivelmente, ocasionando um maior acúmulo de açúcares nos frutos fixados (Moreira et al., 2014).

Em estudo acerca da aplicação de doses crescentes do bioestimulante sobre cachos de uva 'Niágara Rosada' observou-se a diminuição linear do teor de sólidos solúveis dos frutos até a concentração máxima de $20 \mathrm{mg} \mathrm{L}^{-1}$ (Tecchio et al., 2006), resultado contrário ao obtido no presente estudo. Enquanto que, a aplicação do bioestimulante em tomates 'Giuliana' não surtiram efeitos sobre as características agronômicas dos frutos, em comparação ao tratamento controle (Ramos, 2013), demonstrando que a ação dos fitormônios pode depender da espécie em que estes são utilizados.

Destaca-se o ineditismo dos resultados obtidos no presente estudo. Diversos estudos têm abordado a utilização de bioestimulantes em culturas de interesse agronômico. No entanto, para cucurbitáceas esses trabalhos abordam a utilização destes produtos, a base de fitormônios, principalmente na fase vegetativa (Junglaus, 2007) ou mesmo sobre a forma de tratamento de sementes (Silva et al., 2014), não havendo estudos acerca da aplicação direta sobre frutos dessa família.

Assim, demonstra-se que a aplicação do bioestimulante diretamente sobre os frutos consta como uma técnica a ser explorada na produção de melões, podendo ser submetida a novos estudos visando sua utilização em outras espécies. 


\section{CONCLUSÕES}

A aplicação de bioestimulante, até a concentração de $20 \mathrm{~mL} \mathrm{~L}^{-1}$, eleva o teor de sólidos solúveis em frutos de melão rendilhado.

Conflito de interesses: o manuscrito foi preparado e revisado com a participação de todos os autores, que declaram não ter qualquer conflito de interesses que possa afetar a validade dos resultados do trabalho apresentado.

\section{REFERÊNCIAS BIBLIOGRÁFICAS}

Agrofit. 2017. Sistema de agrotóxicos fitossanitários. Em: http://agrofit.agricultura.gov.br/agrofit_cons/principal_agrofit_cons; consulta: abril 2017.

Chiavegato, E.J. e V.T. Lima. 2007. Efeito de Stimulate 10X em diferentes doses e estádios de aplicação via foliar e tratamento de sementes em algodoeiro. p. 1. Em: Livro de resumos, Congresso Brasileiro de Algodão. Abrapa, Amipa e Embrapa. Uberlândia, Brasil.

Donagemma G.K., V.D.B. Campos, S.B. Calderano, W.G. Teixeira e J.H.M. Viana. 2011. Manual de métodos de análise de solo. $2^{\mathrm{a}}$. ed. Brasileira de Pesquisa Agropecuária, Rio de Janeiro, Brasil.

IBGE. 2014. Produção agrícola municipal. Em: http://www. sidra.ibge.gov.br, consulta: outubro 2016.

Izidório, T.H.C., S.F. Lima, E.P. Vendruscolo, J. Ávila e R.C.F. Alvarez. 2015. Bioestimulante via foliar em alface após o transplantio das mudas. Rev. Agric. Neotrop. 2(2), 49-56.

Junglaus, R.W. 2007. Aplicação de bioestimulante vegetal sobre o desenvolvimento de pepineiro (Cucumis sativus) enxertado e não enxertado. Dissertação de Mestrado. Universidade do Estado de São Paulo, Botucatu, Brasil.

Medeiros, J.D., S.C.L. Santos, M.J.T. Câmara e M.Z.D. Negreiros. 2007. Produção de melão Cantaloupe influenciado por coberturas do solo, agro têxtil e lâminas de irrigação. Hortic. Bras. 25(4), 538-543. Doi: 10.1590/ S0102-05362007000400009

Medeiros, L.S., P.V. Ferreira, I.D.E. De Carvalho, F.S. Oliveira e J. Silva. 2015. Primeiro ciclo de seleção massal na população PM3 de melão (Cucumis melo L.). Rev. Verde 10(4), 21-27. Doi: 10.18378/rvads.v10i4.3473

Moreira, R.A., M.C.M. Cruz, M.C.P. Fagundes, L.A. Pantoja e A.S. Santos. 2014. Carboidratos foliares durante a floração e estádios iniciais de crescimento de frutilhos em tagerineira ‘Ponkan'. Pesq. Agropec. Bras. 49(1), 3439. Doi: 10.1590/S0100-204X2014000100005

Pereira, M.C.T., J.H. Crane, S. Nietsche, W. Montas e M.A. Santos. 2014. Growth regulators on fruit set and quality of parthenocarpic fruit in atemoya 'Gefner'. Pesq. Agropec. Bras. 49(4), 281-289. Doi: 10.1590/ S0100-204X2014000400006

Prado, A.K., E.C. Machado, C.L. Medina, D.F.S.P Machado e P. Mazzafera. 2007. Florescimento e frutificação em laranjeiras 'Valência' com diferentes cargas de frutos e submetidas ou não à irrigação. Bragantia 66(2),173182. Doi: 10.1590/S0006-87052007000200001

Ramos A.R.P. 2013. Produtos de efeitos fisiológicos no desenvolvimento de plantas de tomate 'Giuliana', na produção e pós-colheita de frutos. Tese de doutorado. Universidade do Estado de São Paulo, Botucatu, Brasil.

Sales Júnior, R., S.P.F. Soares, J. Amaro Filho, G.H.S. Nunes e V.S. Miranda. 2004. Qualidade do melão exportado pelo porto de Natal. Hortic. Bras., 22(1), 98-100. Doi: 10.1590/S0102-05362004000100020

Santos H.G., P.K.T. Jacomine, L.H.C. Anjos, V.A. Oliveira, J.F. Lumbreiras, M.R. Coelho, J.A. Almeida, T.J.F. Cunha e J.B. Oliveira. 2013. Sistema brasileiro de classificação de solos. $3^{a}$. ed. Empresa Brasileira de Pesquisa Agropecuária, Brasília, Brasil.

Silva, M.J.R., A.C.B. Bolfarini, L.F.O.S. Rodrigues, E.O. Ono e J.D. Rodrigues. 2014. Formação de mudas de melancia em função de diferentes concentrações e formas de aplicação de bioestimulante. Sci. Plena 10(10),1-9.

Sobrinho, R.B., J.A. Guimarães, J.A.D. Freitas e D. Terao. 2008. Produção integrada de melão. Embrapa Agroindústria Tropical e Banco do Nordeste do Brasil, Fortaleza, Brasil.

Taiz, L., E. Zeiger, I.M. Moller e A. Murphy. 2017. Fisiologia e desenvolvimento vegetal. $6^{\mathrm{a}}$. ed. Artmed, Porto Alegre, Brasil.

Talón, M., F.R. Tadeo, W. Ben-Cheikh, A. Gomes-Cadenas, J. Mehouachi, J. Pérez-Botella e E. Primo-Millo. 1998. Hormonal regulation of fruits set and abcission in citrus: classical concepts and new evidence. Acta. Hortic, 463, 209-218. Doi: 10.17660/ActaHortic.1998.463.24

Tavares, S., P.R. Camargo, E.J. Ambrosano, S.C. Cato e D.E. Foltran. 2015. Efeitos de bioestimulante no desenvolvimento de frutos de tomateiro 'Carmen'. Cad. Agroecol., 9(4), 1-10.

Tecchio, M.A., S. Leonel, E. Camilis, G.C. Moreira, E. Pires e J.D. Rodrigues. 2006. Uso de bioestimulante na videira Niagara Rosada. Ciênc. Agrotec. 30(6), 1236-1240. Doi: 10.1590/S1413-70542006000600030

Vendruscolo, E.P., H.B. Souza, L.A. Arruda, S.F. Lima e R.C.F. Alvarez. 2015. Biorregulador na germinação e desenvolvimento inicial do algodoeiro. Rev. Ciênc. Agro-amb. 13(2), 32-40.

Vieira, E.L. e P.R.C. Castro. 2001. Ação de bioestimulante na germinação de sementes, vigor de plântulas, crescimento radicular e produtividade de soja. J. Seed Sci. 23(2), 222-228. Doi: 10.1590/S0034-737X2011000500017 